Ciência e Natura, Santa Maria, v. 37 Ed. Especial PROFMAT, 2015, p. 89 - 94

Revista do Centro de Ciências Naturais e Exatas - UFSM

ISSN impressa: 0100-8307 ISSN on-line: 2179-460X

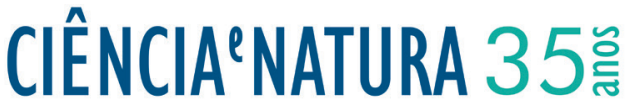

\section{Um irracional: o número de Euler}

\section{An irrational: Euler's number}

\author{
Paulo Leandro Dattori da Silva ${ }^{* 1}$ e Silvana de Lourdes Gálio Spolaor ${ }^{2}$ \\ 1,2 Instituto de Ciências Matemáticas e de Computação - Univesidade de São Paulo - USP, SP, Brasil
}

\begin{abstract}
Resumo
O conjunto dos números reais, $\mathbb{R}$, é um tema delicado de ser tratado na sala de aula. Em particular, o estudo do conjunto dos números irracionais fica prejudicado pela falta de exemplos encontrada em muitos livros didáticos. O principal objetivo deste trabalho é apresentar de maneira clara um importante exemplo de número irracional: o número de Euler. Aqui apresentaremos a motivação para o surgimento do número de Euler, bem como, a demonstração de sua irracionalidade.
\end{abstract}

Palavras-chave: números reais, número de Euler, sequências, séries.

\begin{abstract}
The set of real numbers, $\mathbb{R}$, is a delicate issue to be addressed in the classroom. In particular, the study of the set of irrational numbers is hampered by lack of examples found in many textbooks. The main goal of this paper is presenting of clear manner an important example of irrational number: Euler's number. Here we present the motivation for the Euler's number as well, the proof of their irrationality.
\end{abstract}

Keywords: real numbers, Euler's number, sequence, serie. 


\section{Introdução}

O conjunto dos números reais, $\mathbb{R}$, é um assunto delicado tanto para quem quer aprender quanto para quem quer ensinar. A dificuldade é ainda maior quando lidamos com números que não são racionais. Não é uma tarefa difícil verificar que existem números que não são racionais. Por exemplo, se tomarmos dois quadrados de área 1 e dividirmos cada um deles na diagonal, de comprimento $d$, teremos quatro triângulos. Podemos, então, usar esses triângulos para forma um quadrado de lado $d$ e área 2. Logo, $d^{2}=2$. Tal $d$ é denotado por $\sqrt{2}$.

Segue facilmente de propriedades de números pares que $\sqrt{2} \notin \mathbb{Q}$. Então, eis aqui um primeiro (e simples) exemplo de um número não racional.

Usualmente denotamos $\mathbb{R}=\mathbb{Q} \cup(\mathbb{R}-\mathbb{Q})$, sendo $\mathbb{R}-\mathbb{Q}$ o conjunto dos números irracionais (não vazio, pois $\sqrt{2} \in \mathbb{R}-\mathbb{Q}$ ).

Fazendo uso de um pouco mais de propriedades de divisibilidade de números primos é possível verificar que se $p$ é primo então $\sqrt{p}$ é irracional. De modo mais geral, se $p$ é primo e $r \in \mathbb{Q}$ então $r \sqrt{p} \notin \mathbb{Q}$. Logo, temos aqui infinitos (enumeráveis) exemplos de números irracionais.

Defendemos que os exemplos mencionados acima podem (e devem) ser apresentados aos alunos do ensino básico (respeitado o período adequado). Porém, nem todo número irracional é da forma $r \sqrt{p}$, com $p$ primo e $r \in \mathbf{Q}$.

Um importante exemplo de tal número irracional que aparece nos livros de ensino médio é o número $e$, conhecido como número de Euler. Leonhard Euler (1707-1783), matemático suíço, foi um dos primeiros a estudar as propriedades deste número. Porém, segundo Maor (2008), as origens de $e$ são anteriores a Euler e aparentemente surgiram em problemas de juros.

Acreditamos que seja possível discutir a demonstração da irracionalidade de $e$ com os alunos do ensino médio. De fato, podemos definir $e$, e verificar sua irracionalidade, utilizando apenas propriedades de sequências e séries.

Neste texto apresentaremos uma motivação para o surgimento de $e$, bem como, sua definição. Também, apresentaremos uma demonstração detalhada da irracionalidade de $e$, baseada naquela apresentada em Maor (2008). Optamos por uma abordagem menos técnica e mais intuitiva na tentativa de tornar o texto mais agradável e acessível.

Nosso objetivo é que este texto seja utilizado por Professores na elaboração de um conjunto de aulas a respeito de números irracionais.

\section{Motivação e Definição}

Não se sabe a data exata do surgimento do número $e$, segundo Maor (2008):

As origens de e não são tão claras, elas parecem recuar ao século XVI, quando se percebeu que a expressão $(1+1 / n)^{n}$, que aparecia na fórmula dos juros compostos, tendia a um certo limite - cerca de 2,71828 - à medida que $n$ aumenta. Assim, tornou-se o primeiro número a ser definido por um processo de limite, $e=\lim _{n \rightarrow \infty}(1+1 / n)^{n}$.

O número $e$ parece surgir de forma espontânea em muitas situações práticas. Vejamos um exemplo utilizando juros.

Um capital de $\mathrm{R} \$ 100,00$ é aplicado a uma taxa de $15 \%$ ao longo de um ano. Vamos imaginar que o juro seja incorporado apenas ao final do ano. Neste caso teríamos um montante equivalente a

$$
100+\frac{15}{100} 100, \quad \text { isto é, } \quad \mathrm{R} \$ 115,00 .
$$

Isto significa que o capital ficou estagnado durante todo $\mathrm{o}$ ano e apenas na passagem para o ano seguinte sofreu o acréscimo de $15 \%$.

Note que se desejássemos sacar os $\mathrm{R} \$ 100,00$ ao final de 6 meses não receberíamos nada de juros. Então, parece mais razoável que ao sacar os $R \$ 100,00$ ao final de 6 meses recebessemos $\frac{15}{2} \%$ de juros. Dessa forma, ao final de 6 meses o montante passaria a valer $100+\frac{15}{200} 100$, isto é, $R \$ 107,50$ ao invés de $R \$ 100,00$. Logo, ao deixar este montante aplicado até o final do ano ele sofreria um acréscimo de $\frac{15}{2} \%$ e seu valor passaria a ser $100+\frac{15}{200} 100+\frac{15}{200}\left(100+\frac{15}{200} 100\right)$, o que equivale a $\left(100+\frac{15}{200} 100\right)\left(1+\frac{15}{200}\right) ;$ ou ainda, $100\left(1+\frac{15}{200}\right)\left(1+\frac{15}{200}\right)$. Assim, se a taxa for distribuída a cada 6 meses, no final de um ano o capital passaria a ser $100\left(1+\frac{15}{200}\right)^{2}$.

Podemos aplicar o raciocínio acima para verificar o rendimento do capital a cada 4 meses, isto é, a cada trimestre. No final do primeiro trimestre deveríamos ter $100+\frac{15}{400} 100$, ou ainda, $100\left(1+\frac{15}{400}\right)$. No final do segundo trimestre o capital deveria ser igual a $100\left(1+\frac{15}{400}\right)^{2}$. No final do terceiro trimestre deveríamos ter $100\left(1+\frac{15}{400}\right)^{3}$ e, finalmente, no término do 
ano o capital passaria a ser $100\left(1+\frac{15}{400}\right)^{4}$.

Repetindo o raciocínio anterior, se imaginarmos a taxa distribuída uniformemente ao longo do ano dividido em $n$ períodos, no final do ano teríamos

$$
C=100\left(1+\frac{15}{100 n}\right)^{n} .
$$

Aparentemente, os bancos usam $n=12$.

Surge, então, uma pergunta natural: a medida que $n$ aumenta, qual o valor de $\left(1+\frac{15}{100 n}\right)^{n}$ ?

Podemos simplificar nossa pergunta fazendo a mudança $\frac{15}{100 n}=\frac{1}{m}$ ou, equivalentemente, $100 n=15 m$; note que $n$ aumenta se, e somente se, $m$ aumenta.

Assim, podemos escrever

$$
\left(1+\frac{15}{100 n}\right)^{n}=\left(1+\frac{1}{m}\right)^{\frac{15 m}{100}}=\left[\left(1+\frac{1}{m}\right)^{m}\right]^{\frac{15}{100}} .
$$

Nossa pergunta, então, é equivalente a seguinte: a medida que $m$ aumenta, qual o valor de $\left(1+\frac{1}{m}\right)^{m}$ ?

Antes de responder a pergunta acima, vamos estudar o comportamento da sequência $\left(S_{n}\right)$, cujos termos são dados por

$$
S_{n}=\frac{1}{0 !}+\frac{1}{1 !}+\frac{1}{2 !}+\cdots+\frac{1}{n !} .
$$

Note que $1 \leq S_{n}<S_{n+1}$ para todo $n$.

Também, note que podemos escrever $S_{n}$ na forma

$$
S_{n}=\sum_{k=0}^{n} \frac{1}{k !} .
$$

Logo, quando fazemos $n$ aumentar indefinidamente (ou seja, $n$ ir para o infinito) queremos calcular

$$
\sum_{k=0}^{\infty} \frac{1}{k !}
$$

isto é, queremos encontrar $S \in \mathbb{R}$ tal que

$$
S=\sum_{k=0}^{\infty} \frac{1}{k !} .
$$

Note que, para $n \geq 3$, temos $n !=1.2 .3 \cdots n>$ 1.2.2 $\cdots 2=2^{n-1}$. Assim, podemos escrever

$$
S_{n}<1+1+\frac{1}{2}+\frac{1}{2^{2}}+\cdots+\frac{1}{2^{n-1}}=1+\sum_{k=0}^{n-1} \frac{1}{2^{k}} .
$$

Daí,

$$
S_{n}<1+\sum_{k=0}^{n-1} \frac{1}{2^{k}}<1+\sum_{k=0}^{\infty} \frac{1}{2^{k}}
$$

Como $\sum_{k=0}^{\infty} \frac{1}{2^{k}}=1+\frac{1}{2}+\frac{1}{2^{2}}+\cdots+\frac{1}{2^{k}}+\cdots$ é uma Progressão Geométrica com primeiro termo igual a $1 \mathrm{e}$ razão igual a $\frac{1}{2}$, podemos aplicar a fórmula para calcular a soma de uma PG infinita e, então, obtemos

$$
\sum_{k=0}^{\infty} \frac{1}{2^{k}}=\frac{1}{1-\frac{1}{2}}=\frac{1}{\frac{1}{2}}=2 .
$$

Logo, para cada $n$ temos $S_{n}<3$. Assim, mostramos que a sequência $\left(S_{n}\right)$ é limitada superiormente por 3, ou seja, seus valores nunca excedem 3 .

Portanto, como $S_{n}<S_{n+1}$ e $S_{n}$ está entre 1 e 3, para cada $n \geq 0$, deve existir um $S \in \mathbb{R}$, com $1 \leq S \leq 3$, tal que

$$
S=\sum_{k=0}^{\infty} \frac{1}{k !} .
$$

Abaixo, segue uma tabela que nos sugere uma aproximação para a soma $S$ :

Tabela 1: Aproximação de $S$

\begin{tabular}{|c|l|c|}
\hline$n$ & $S_{n}=\sum_{k=0}^{n} \frac{1}{k !}$ & $S_{n}$ \\
\hline 0 & $\frac{1}{1}$ & 1 \\
\hline 1 & $\frac{1}{1}+\frac{1}{1}$ & 2 \\
\hline 2 & $\frac{1}{1}+\frac{1}{1}+\frac{1}{2}$ & 2,5 \\
\hline 3 & $\frac{1}{1}+\frac{1}{1}+\frac{1}{2}+\frac{1}{6}$ & $\approx 2,666666$ \\
\hline 4 & $\frac{1}{1}+\frac{1}{1}+\frac{1}{2}+\frac{1}{6}+\frac{1}{24}$ & $\approx 2,708332$ \\
\hline 5 & $\frac{1}{1}+\frac{1}{1}+\frac{1}{2}+\frac{1}{6}+\frac{1}{24}+\frac{1}{120}$ & $\approx 2,716665$ \\
\hline 6 & $\frac{1}{1}+\frac{1}{1}+\frac{1}{2}+\frac{1}{6}+\frac{1}{24}+\frac{1}{120}+\frac{1}{720}$ & $\approx 2,718053$ \\
\hline
\end{tabular}

Pela tabela 1 podemos indicar que $S$ é aproximadamente 2,718 .

Agora, vamos mostrar que quando $n$ aumenta $T_{n}=$ $\left(1+\frac{1}{n}\right)^{n}$ fica perto de $S$.

Recordemos que o binômio de Newton se escreve como

$$
(a+b)^{n}=\left(\begin{array}{c}
n \\
0
\end{array}\right) a^{n} b^{0}+\left(\begin{array}{c}
n \\
1
\end{array}\right) a^{n-1} b^{1}+\cdots+\left(\begin{array}{l}
n \\
n
\end{array}\right) a^{0} b^{n},
$$


sendo $\left(\begin{array}{l}n \\ k\end{array}\right)=\frac{n !}{k !(n-k) !}$. Então, para $a=1$ e $b=\frac{1}{n}$, temos:

$$
\begin{aligned}
T_{n}= & \left(1+\frac{1}{n}\right)^{n} \\
= & \left(\begin{array}{l}
n \\
0
\end{array}\right) 1^{n}\left(\frac{1}{n}\right)^{0}+\left(\begin{array}{c}
n \\
1
\end{array}\right) 1^{n-1}\left(\frac{1}{n}\right)^{1} \\
& \quad+\left(\begin{array}{c}
n \\
2
\end{array}\right) 1^{n-2}\left(\frac{1}{n}\right)^{2}+\cdots+\left(\begin{array}{l}
n \\
n
\end{array}\right) 1^{0}\left(\frac{1}{n}\right)^{n} \\
= & 1+n \cdot \frac{1}{n}+\frac{n(n-1)}{2 !} \cdot \frac{1}{n^{2}}+\cdots \\
\quad & \quad \frac{n(n-1)(n-2) \cdots 1}{n !} \cdot \frac{1}{n^{n}} \\
= & 1+1+\left(1-\frac{1}{n}\right) \cdot \frac{1}{2 !}+\cdots \\
& \quad+\left(1-\frac{1}{n}\right) \cdot\left(1-\frac{2}{n}\right) \cdots\left(1-\frac{n-1}{n}\right) \cdot \frac{1}{n !} .
\end{aligned}
$$

Observe que as expressões dentro de cada parênteses (na última linha acima) são menores do que 1 ; assim $T_{n} \leq S_{n}$. Então, podemos concluir que $T_{n}<3$ qualquer que seja $n$. Afirmamos que a sequência $\left(T_{n}\right)$ é crescente. De fato,

$$
\begin{aligned}
T_{n}=1+ & +\left(1-\frac{1}{n}\right) \cdot \frac{1}{2 !}+\cdots \\
& +\left(1-\frac{1}{n}\right) \cdot\left(1-\frac{2}{n}\right) \cdots\left(1-\frac{n-1}{n}\right) \cdot \frac{1}{n !}
\end{aligned}
$$

e

$$
\begin{aligned}
T_{n+1}= & 1+1+\left(1-\frac{1}{n+1}\right) \cdot \frac{1}{2 !} \\
+ & \left(1-\frac{1}{n+1}\right) \cdot\left(1-\frac{2}{n+1}\right) \cdot \frac{1}{3 !}+\cdots \\
+ & \left(1-\frac{1}{n+1}\right) \cdot\left(1-\frac{2}{n+1}\right) \\
& \ldots\left(1-\frac{n-1}{n+1}\right) \cdot \frac{1}{n !} \\
+ & \left(1-\frac{1}{n+1}\right) \cdot\left(1-\frac{2}{n+1}\right) \\
& \quad \cdots\left(1-\frac{n-1}{n+1}\right) \cdot\left(1-\frac{n}{n+1}\right) \cdot \frac{1}{(n+1) !} .
\end{aligned}
$$

Como a última parcela de $T_{n+1}$ é maior que zero, temos

$$
\begin{aligned}
T_{n+1} & >1+1+\left(1-\frac{1}{n+1}\right) \cdot \frac{1}{2 !} \\
& +\left(1-\frac{1}{n+1}\right) \cdot\left(1-\frac{2}{n+1}\right) \cdot \frac{1}{3 !}+\cdots \\
& +\left(1-\frac{1}{n+1}\right) \cdots\left(1-\frac{n-1}{n+1}\right) \cdot \frac{1}{n !}
\end{aligned}
$$

Agora,

$$
\begin{aligned}
n<n+1 & \Leftrightarrow \frac{1}{n}>\frac{1}{n+1} \Leftrightarrow-\frac{1}{n}<-\frac{1}{n+1} \\
& \Leftrightarrow-\frac{k}{n}<-\frac{k}{n+1}, \quad \forall k>0 \\
& \Leftrightarrow 1-\frac{k}{n}<1-\frac{k}{n+1}, \quad \forall k>0 .
\end{aligned}
$$

Em particular, tomando $k=1,2, \cdots, n-1$, temos

$$
\begin{aligned}
T_{n+1}>1+1+\left(1-\frac{1}{n+1}\right) \cdot \frac{1}{2 !} & \\
& +\left(1-\frac{1}{n+1}\right) \cdot\left(1-\frac{2}{n+1}\right) \cdot \frac{1}{3 !}+\cdots \\
& +\left(1-\frac{1}{n+1}\right) \cdot\left(1-\frac{2}{n+1}\right) \cdots\left(1-\frac{n-1}{n+1}\right) \cdot \frac{1}{n !} \\
> & 1+1+\left(1-\frac{1}{n}\right) \cdot \frac{1}{2 !}+\cdots+\left(1-\frac{n-1}{n}\right) \cdot \frac{1}{n !}=T_{n} .
\end{aligned}
$$

Logo, $T_{n+1}>T_{n}$.

Portanto, como $\left(T_{n}\right)$ é uma seqüência crescente e cada termo $T_{n}$ está entre 2 e 3 , deve existir $T \in \mathbb{R}$, com $2 \leq T \leq 3$, tal que $T_{n}$ se aproxima de $T$ a medida que $n$ aumenta.

Abaixo, segue uma tabela que nos sugere uma aproximação para o valor de $T$ :

Tabela 2: Aproximação de $T$

\begin{tabular}{|c|l|c|}
\hline$n$ & $\left(1+\frac{1}{n}\right)^{n}$ & $T_{n}$ \\
\hline 1 & $\left(1+\frac{1}{1}\right)^{1}$ & 2 \\
\hline 2 & $\left(1+\frac{1}{2}\right)^{2}$ & 2,25 \\
\hline 10 & $\left(1+\frac{1}{10}\right)^{10}$ & $\approx 2,59374$ \\
\hline 100 & $\left(1+\frac{1}{100}\right)^{100}$ & $\approx 2,70481$ \\
\hline 1.000 & $\left(1+\frac{1}{1.000}\right)^{1.000}$ & $\approx 2,71692$ \\
\hline 10.000 & $\left(1+\frac{1}{10.000}\right)^{10.000}$ & $\approx 2,71814$ \\
\hline 100.000 & $\left(1+\frac{1}{100.000}\right)^{100.000}$ & $\approx 2,71826$ \\
\hline 1.000 .000 & $\left(1+\frac{1}{1.000 .000}\right)^{1.000 .000}$ & $\approx 2,71828$ \\
\hline 10.000 .000 & $\left(1+\frac{1}{10.000 .000}\right)^{10.000 .000}$ & $\approx 2,71828$ \\
\hline
\end{tabular}

Pela tabela 2 podemos indicar que $T$ é aproximadamente 2,718 . 
Vamos mostrar que de fato $S=T$. Para isso, vamos mostrar que $S \geq T$ e $S \leq T$.

Para mostrar que $S \geq T$ é simples, pois como $T_{n} \leq$ $S_{n} \leq S$ temos $T \leq S$.

Vamos mostrar, agora, que $S \leq T$. Fixe $m$ e seja $n$ tal que $m<n$. Denotemos por $R_{m, n}$ os primeiros $m+1$ termos de $T_{n}$, isto é,

$$
\begin{aligned}
R_{m, n}= & 1+1+\left(1-\frac{1}{n}\right) \cdot \frac{1}{2 !}+\cdots \\
& +\left(1-\frac{1}{n}\right) \cdot\left(1-\frac{2}{n}\right) \cdot \ldots \cdot\left(1-\frac{m-1}{n}\right) \cdot \frac{1}{m !} .
\end{aligned}
$$

Note que, como $m<n$ e todos os termos de $\left(T_{n}\right)$ são positivos, $R_{m, n}<T_{n}$. Note também que quando $n$ aumenta $R_{m, n}$ fica perto de $S_{m}$, pois quando $n$ aumenta $1-\frac{1}{n}, 1-\frac{2}{n}, \cdots, 1-\frac{m-1}{n}$ ficam perto de 1 .

Agora, como $R_{m, n}<T_{n} \leq T$, temos $S_{m} \leq T$ qualquer que seja $m$.

Logo,

$$
S=\sum_{k=0}^{\infty} \frac{1}{k !} \leq T \text {. }
$$

Portanto, $S=T$.

Definição: Definimos $e=\sum_{n=0}^{\infty} \frac{1}{n !}$.

Também, $e=\lim _{n \rightarrow \infty}\left(1+\frac{1}{n}\right)^{n}$ e lê-se

"limite de $\left(1+\frac{1}{n}\right)^{n}$ quando $n$ tende ao infinito é igual a $e^{\prime}$.

Finalmente, estamos prontos para responder nossa a pergunta: a medida que $n$ aumenta, qual é o valor de $\left(1+\frac{15}{100 n}\right)^{n} ?$

Resposta: $e^{\frac{15}{100}}$.

Portanto, um capital de $\mathrm{R} \$ 100,00$ aplicado a uma taxa de $15 \%$ ao longo de um ano deveria ser

$$
\mathrm{R} \$ 100 e^{\frac{15}{100}} \approx \mathrm{R} \$ 116,00
$$

ao final da aplicação. Compare (1) e (3).

A argumentação acima pode ser estendida a qualquer capital $C_{0}$ aplicado a uma taxa de $t \%(t \in \mathbb{Q})$. Ao final de um ano o montante deveria ser

$$
\mathrm{C}_{0} e^{\frac{t}{100}} \text {. }
$$

Nossa argumentação mostra que a uma taxa de 15\% tem-se diferença ao calcularmos os juros por (1) ou por (4). De fato, usando (4) podemos verificar que para $t$ pequeno tanto faz aplicar (1) ou (4), pois vamos obter o mesmo montante final. Porém, para $t$ grande (4) fornece um montante maior.
Pelas tabelas 1 e 2 somos levados a concluir que $2<e<3$. Vamos verificar que de fato isto ocorre. Evidentemente, $2<\sum_{n=0}^{\infty} \frac{1}{n !}$. Além disso,

$$
\sum_{n=0}^{\infty} \frac{1}{n !}=1+1+\frac{1}{2}+\frac{1}{6}+\sum_{n=4}^{\infty} \frac{1}{n !}
$$

e, como $n !=1.2 .3 \cdots n>1.2 .2 \cdots 2=2^{n-1}$ para $n \geq 3$, temos

$$
\sum_{n=0}^{\infty} \frac{1}{n !} \leq 1+1+\frac{1}{2}+\frac{1}{6}+\sum_{n=4}^{\infty} \frac{1}{2^{n-1}} .
$$

Agora, aplicando a fórmula para calcular a soma de uma PG infinita, temos

$$
\sum_{n=4}^{\infty} \frac{1}{2^{n-1}}=\frac{\frac{1}{2^{3}}}{1-\frac{1}{2}}=\frac{1}{4}
$$

Logo,

$$
\sum_{n=0}^{\infty} \frac{1}{n !} \leq 1+1+\frac{1}{2}+\frac{1}{6}+\frac{1}{4}<1+1+\frac{1}{2}+\frac{1}{4}+\frac{1}{4}=3 .
$$

Portanto, $2<e<3$.

\section{A irracionalidade de $e$}

Em Maor (2008) encontramos uma demonstração da irracionalidade de $e$ que utiliza "apenas" propriedades de séries numéricas. Com o objetivo de tornar este texto o mais auto-sucifiente possível, vamos reproduzir aqui a argumentação; porém, optamos por escrever um texto com mais detalhes.

Suponha, por absurdo, que $e$ é um número racional, ou seja, $e=\frac{p}{q}$, com $p$ e $q$ naturais. Podemos supor $m d c(p, q)=1$. Como $2<e<3$ temos que $e \notin \mathbb{N}$; consequentemente, $q \geq 2$.

Sabemos que

$$
e=\sum_{n=0}^{\infty} \frac{1}{n !}=\frac{1}{0 !}+\frac{1}{1 !}+\frac{1}{2 !}+\frac{1}{3 !}+\cdots .
$$

Assim

$$
\begin{aligned}
\frac{p}{q}=e= & 1+1+\frac{1}{2 !}+\frac{1}{3 !}+\cdots+\frac{1}{(q-1) !}+\frac{1}{q !} \\
& +\frac{1}{(q+1) !}+\cdots+\frac{1}{n !}+\cdots .
\end{aligned}
$$

Multiplicando a igualdade acima por $q$ !, obtemos

$$
\begin{aligned}
\frac{p}{q} \cdot q ! & =1 \cdot q !+1 \cdot q !+\frac{1}{2 !} \cdot q !+\frac{1}{3 !} \cdot q ! \\
& +\cdots+\frac{1}{(q-1) !} \cdot q !+\frac{1}{q !} \cdot q !+\frac{1}{(q+1) !} \cdot q ! \\
& +\cdots+\frac{1}{n !} \cdot q !+\cdots
\end{aligned}
$$


Daí,

$$
\begin{aligned}
\frac{p}{q} \cdot q \cdot(q-1) ! & =q !+q !+\frac{1}{2 !} \cdot 1 \cdot 2 \cdot 3 \cdot \ldots \cdot q \\
& +\frac{1}{3 !} \cdot 1 \cdot 2 \cdot 3 \cdot \ldots \cdot q+\ldots \\
& +\frac{1}{(q-1) !} \cdot q \cdot(q-1) !+\frac{1}{q !} \cdot q ! \\
& +\frac{1}{(q+1) \cdot q !} \cdot q !+\cdots+\frac{1}{n !} \cdot q !+\ldots
\end{aligned}
$$

e, consequentemente,

$$
\begin{aligned}
p \cdot(q-1) ! & =q !+q !+3 \cdot 4 \cdot \ldots \cdot q+4 \cdot 5 \cdot \ldots \cdot q \\
& +\ldots+q+1+\frac{1}{(q+1)} \\
& +\frac{1}{(q+2)(q+1)}+\cdots+\frac{1}{n !} \cdot q !+\ldots
\end{aligned}
$$

Logo,

$$
\begin{aligned}
& p \cdot(q-1) !-(q !+q !+3 \cdot 4 \cdot \ldots \cdot q+\ldots+q+1) \\
& =\frac{1}{(q+1)}+\frac{1}{(q+2)(q+1)}+\ldots+\frac{1}{n !} \cdot q !+\ldots
\end{aligned}
$$

Observe que no lado esquerdo da igualdade (5) as parcelas são números inteiros, enquanto que no lado direito as parcelas são frações; além disso, como em (5) tem-se $q \geq 2$, obtemos que $\frac{1}{q} \leq \frac{1}{2}$. Daí, em (5) temos

$$
\begin{aligned}
\frac{1}{q+1} & \leq \frac{1}{3} \\
\frac{1}{(q+2)(q+1)} & \leq \frac{1}{3} \cdot \frac{1}{3}=\frac{1}{3^{2}} \\
\frac{1}{n !} \cdot q !=\frac{1}{(q+1) \cdot \ldots \cdot(q+(n-q))} & \leq \frac{1}{3^{n-q}} .
\end{aligned}
$$

Logo, em (5), temos

$$
\begin{aligned}
\frac{1}{q+1} & +\frac{1}{(q+2)(q+1)}+\ldots+\frac{1}{n !} \cdot q !+\ldots \\
& \leq \frac{1}{3}+\frac{1}{3^{2}}+\ldots+\frac{1}{3^{n-q}}+\ldots
\end{aligned}
$$

Observe que

$$
\frac{1}{3}+\frac{1}{3^{2}}+\ldots+\frac{1}{3^{n-q}}+\ldots
$$

é uma Progressão Geométrica, sendo que o primeiro termo é $\frac{1}{3}$ e a razão também é $\frac{1}{3}$; logo,

$$
\frac{1}{3}+\frac{1}{3^{2}}+\cdots+\frac{1}{3^{n-q}}+\cdots=\frac{\frac{1}{3}}{1-\frac{1}{3}}=\frac{\frac{1}{3}}{\frac{2}{3}}=\frac{1}{2}
$$

Portanto, segue de (5), (6) e (7) que

$$
p \cdot(q-1) !-(q !+q !+3 \cdot 4 \cdot \ldots \cdot q+\ldots+q+1) \leq \frac{1}{2} .
$$

Absurdo, pois não existe número inteiro entre 0 e $\frac{1}{2}$. $\mathrm{O}$ absurdo ocorreu ao supor que $e$ é número racional; portanto, $e$ é um número irracional.

\section{Referências}

Ávila, G., 2006. Análise para a Licenciatura, $3^{\mathrm{a}}$ edição. São Paulo: Blucher.

Figueiredo, D.G., 1985. Números Irracionais e transcendentes. Sociedade Brasileira de Matemática, Brasília.

Maor, E., 2008. e: A História de um Número, $5^{\text {a }}$ edição. Trad. Jorge Calife. Rio de Janeiro: Editora Record.

Spolaor, S. L. G., 2013. Números irracionais: $\pi$ e $e$. Dissertação de Mestrado (PROFMAT). ICMC/USP. 